\title{
Erratum to: Forward and backward span for verbal and visuo- spatial data: standardization and normative data from an Italian adult population
}

\author{
Marco Monaco - Alberto Costa - Carlo Caltagirone • \\ Giovanni Augusto Carlesimo
}

Published online: 10 December 2014

(C) Springer-Verlag Italia 2014

Erratum to: Neurol Sci (2013) 34:749-754

DOI 10.1007/s10072-012-1130-x

Authors would like to publish an erratum to correct the errors in the tables. The values in the columns under the title Corsi span backward in Tables 3 and 4 are updated and the corrected tables are given below.

The online version of the original article can be found under doi: 10.1007/s10072-012-1130-x.

M. Monaco $(\varangle)$ · A. Costa · C. Caltagirone · G. A. Carlesimo

IRCCS Fondazione Santa Lucia, Clinica Neurologica,

Università di Roma “Tor Vergata”, Via Ardeatina 306,

00179 Rome, Italy

e-mail: m.monaco@hsantalucia.it
A. Costa
e-mail: a.costa@hsantalucia.it
C. Caltagirone
e-mail: c.caltagirone@hsantalucia.it
G. A. Carlesimo
e-mail: memolab@hsantalucia.it 
Table 3 Regression equations and correction grids according to age and education for performance scores on the forward and backward versions of the Digit Span and Corsi Block tasks

\begin{tabular}{|c|c|c|c|c|c|c|c|c|c|c|c|c|c|c|c|}
\hline Age & \multicolumn{2}{|c|}{$20-30$} & $31-40$ & \multirow[t]{2}{*}{$41-50$} & \multirow[t]{2}{*}{$51-60$} & $61-70$ & \multicolumn{2}{|c|}{$71-80$} & $81-90$ & \multicolumn{6}{|l|}{ Total } \\
\hline \multicolumn{14}{|c|}{ Digit span forward } & & \\
\hline Mean & 6.47 & & 6.38 & 6.12 & 5.80 & 5.70 & 5.39 & & & 5.84 & & & & & \\
\hline SD & 0.94 & & 1.09 & 1.15 & 0.95 & 0.92 & 0.86 & & 81 & 1.09 & & & & & \\
\hline $\mathrm{E} \backslash \mathrm{A}$ & 20 & 25 & 30 & 35 & 40 & 45 & 50 & 55 & 60 & 65 & 70 & 75 & 80 & 85 & 90 \\
\hline 3 & -0.09 & -0.04 & 0.01 & 0.07 & 0.13 & 0.19 & 0.26 & 0.34 & 0.43 & 0.53 & 0.65 & 0.79 & 0.96 & 1.17 & 1.48 \\
\hline 5 & -0.23 & -0.18 & -0.13 & -0.07 & -0.01 & 0.05 & 0.12 & 0.20 & 0.29 & 0.39 & 0.51 & 0.65 & 0.82 & 1.03 & 1.34 \\
\hline 8 & -0.39 & -0.35 & -0.29 & -0.24 & -0.18 & -0.11 & -0.04 & 0.04 & 0.13 & 0.23 & 0.35 & 0.49 & 0.65 & 0.87 & 1.18 \\
\hline 13 & -0.61 & -0.56 & -0.51 & -0.45 & -0.39 & -0.32 & -0.25 & -0.17 & -0.08 & 0.02 & 0.13 & 0.27 & 0.44 & 0.66 & 0.96 \\
\hline 17 & $*$ & -0.70 & -0.65 & -0.59 & -0.53 & -0.47 & -0.40 & -0.32 & -0.23 & -0.13 & -0.01 & 0.13 & 0.30 & 0.51 & 0.82 \\
\hline Age & 20 & & $31-40$ & $41-50$ & $51-60$ & $61-70$ & $71-80$ & & -90 & Total & & & & & \\
\hline \multicolumn{16}{|c|}{ Digit span backward } \\
\hline Mean & 5.07 & & 5.16 & 4.68 & 4.66 & 4.15 & 3.92 & & 60 & 4.47 & & & & & \\
\hline SD & 1.25 & & 1.04 & 1.04 & 1.24 & 0.91 & 0.98 & & 67 & 1.19 & & & & & \\
\hline $\mathrm{E} \backslash \mathrm{A}$ & 20 & 25 & 30 & 35 & 40 & 45 & 50 & 55 & 60 & 65 & 70 & 75 & 80 & 85 & 90 \\
\hline 3 & 0.14 & 0.19 & 0.24 & 0.29 & 0.35 & 0.41 & 0.48 & 0.55 & 0.64 & 0.73 & 0.84 & 0.97 & 1.13 & 1.34 & 1.63 \\
\hline 5 & -0.06 & -0.02 & 0.03 & 0.08 & 0.14 & 0.20 & 0.27 & 0.35 & 0.53 & 0.53 & 0.64 & 0.77 & 0.93 & 1.13 & 1.42 \\
\hline 8 & -0.31 & -0.26 & -0.21 & -0.16 & -0.10 & -0.04 & 0.02 & 0.10 & 0.19 & 0.28 & 0.39 & 0.52 & 0.68 & 0.89 & 1.18 \\
\hline 13 & -0.62 & -0.58 & -0.53 & -0.48 & -0.42 & -0.36 & -0.29 & -0.21 & -0.13 & -0.03 & 0.08 & 0.21 & 0.37 & 0.57 & 0.86 \\
\hline 17 & $*$ & -0.79 & -0.74 & -0.69 & -0.63 & -0.57 & -0.50 & -0.42 & -0.34 & -0.24 & -0.13 & 0.00 & 0.16 & 0.36 & 0.65 \\
\hline Age & 20 & & $31-40$ & $41-50$ & $51-60$ & $61-70$ & $71-80$ & & -90 & Total & & & & & \\
\hline \multicolumn{16}{|c|}{ Corsi span forward } \\
\hline Mean & 6.00 & & 5.94 & 5.50 & 5.56 & 5.17 & 5.02 & & 42 & 5.38 & & & & & \\
\hline SD & 1.09 & & 1.06 & 1.02 & 1.02 & 0.98 & 0.75 & & 39 & 1.09 & & & & & \\
\hline $\mathrm{E} \backslash \mathrm{A}$ & 20 & 25 & 30 & 35 & 40 & 45 & 50 & 55 & 60 & 65 & 70 & 75 & 80 & 85 & 90 \\
\hline 3 & 0.00 & 0.05 & 0.10 & 0.16 & 0.22 & 0.28 & 0.35 & 0.43 & 0.51 & 0.61 & 0.72 & 0.86 & 1.02 & 1.23 & 1.53 \\
\hline 5 & -0.16 & -0.11 & -0.06 & -0.01 & 0.05 & 0.11 & 0.18 & 0.26 & 0.35 & 0.44 & 0.56 & 0.69 & 0.85 & 1.07 & 1.36 \\
\hline 8 & -0.35 & -0.31 & -0.26 & -0.20 & -0.14 & -0.08 & -0.01 & 0.07 & 0.15 & 0.25 & 0.36 & 0.50 & 0.66 & 0.87 & 1.17 \\
\hline 13 & -0.61 & -0.56 & -0.51 & -0.46 & -0.40 & -0.33 & -0.26 & -0.19 & -0.10 & 0.00 & 0.11 & 0.24 & 0.41 & 0.62 & 0.91 \\
\hline 17 & * & -0.73 & -0.68 & -0.63 & -0.57 & -0.50 & -0.43 & -0.36 & -0.27 & -0.17 & -0.06 & 0.07 & 0.24 & 0.45 & 0.74 \\
\hline Age & 20 & & $31-40$ & $41-50$ & $51-60$ & $61-70$ & $71-80$ & & -90 & Total & & & & & \\
\hline \multicolumn{16}{|c|}{ Corsi span backward } \\
\hline Mean & 5.24 & & 5.38 & 4.70 & 5.04 & 4.66 & 4.43 & & 50 & 4.72 & & & & & \\
\hline SD & 0.90 & & 1.14 & 0.91 & 1.05 & 0.95 & 0.84 & & 00 & 1.13 & & & & & \\
\hline \multirow[t]{2}{*}{ Age } & 20 & 25 & 30 & 35 & 40 & 45 & 50 & 55 & 60 & 65 & 70 & 75 & 80 & 85 & 90 \\
\hline & -0.72 & -0.65 & -0.58 & -0.50 & -0.42 & -0.32 & -0.23 & -0.1 & 0.01 & 0.15 & 0.31 & 0.50 & 0.73 & 1.03 & 1.46 \\
\hline
\end{tabular}

Best linear model: $5.84+1.74 *(\log (100-$ age $)-1.61)+0.28 *(\sqrt{ }$ school -3.28$)$

Significance: age $t=7.19, p<0.025$; school $t=3.60, p<0.025$

Best linear model: $4.47+1.65 *(\log (100-$ age $)-1.61)+0.41 *(\sqrt{ }$ school -3.28$)$

Significance: age $t=6.23, p<0.025$; school $t=4.88, p<0.025$

Best linear model: $5.38+1.69 *(\log (100-$ age $)-1.61)+0.33 *(\sqrt{ }$ school -3.28$)$

Significance: age $t=6.98, p<0.025$; school $t=4.28, p<0.025$

Best linear model: $4.72+2.41 *(\log (100-$ age $)-1.61)$

Significance: age $t=11.04, p<0.025$ 
Table 4 Equivalent scores for performance on the forward and backward versions of both the Digit span and Corsi block tasks and for the ratio between the scores on the backward and forward versions of each task

\begin{tabular}{|c|c|c|c|c|c|}
\hline & \multicolumn{5}{|c|}{ Equivalent scores } \\
\hline & 0 & 1 & 2 & 3 & 4 \\
\hline Percentage of sample distribution below the reported value & 2.90 & 10.40 & 26.40 & 50 & 100 \\
\hline Digit span forward & $<4.26$ & $<4.60$ & $<5.29$ & $<5.75$ & $>5.75$ \\
\hline Digit span backward & $<2.65$ & $<3.29$ & $<3.79$ & $<4.33$ & $>4.33$ \\
\hline Corsi span forward & $<3.46$ & $<4.29$ & $<4.80$ & $<5.37$ & $>5.37$ \\
\hline Corsi span backward & $<3.17$ & $<3.45$ & $<4.09$ & $<4.63$ & $>4.63$ \\
\hline Ratio digit span & $<0.48$ & $<0.58$ & $<0.66$ & $<0.79$ & $>0.79$ \\
\hline Ratio Corsi span & $<0.53$ & $<0.64$ & $<0.77$ & $<0.90$ & $>0.90$ \\
\hline
\end{tabular}

